\title{
Efectos de un procedimiento instruccional en el desarrollo de habilidades para el cuidado de las mamas
}

\author{
Effects of an instructional procedure \\ to develop breast care skills
}

\author{
Jesús Ernesto Valencia, Karla Fabiola Acuña, Juan José Irigoyen \\ y Miriam Yerith Jiménez ${ }^{1}$
}

\begin{abstract}
RESUMEN
Las enfermedades crónicas no transmisibles son la principal causa de muerte en el mundo; en particular, el cáncer de mama es el tipo de cáncer más común entre las mujeres. Los programas implementados se han enfocado en la distribución masiva de información, pero esta no parece ser una estrategia efectiva para modificar las prácticas poco saludables. El modelo psicológico de la salud biológica permite identificar los factores psicológicos que intervienen en el proceso de salud-enfermedad. El objetivo del presente estudio fue evaluar un procedimiento instruccional para entrenar a mujeres con el propósito de que logren identificar y poner en práctica conductas instrumentales relacionadas con el cuidado de las mamas y evaluar su efecto en la calidad de vida de aquéllas. Se diseñó un estudio pretest-postest en el que participaron 22 mujeres, las cuales se asignaron aleatoriamente a dos condiciones de entrenamiento: materiales con texto, video, imágenes y un modelo de simulación, y materiales con texto, video e imágenes. Asimismo, se aplicaron dos cuestionarios: uno elaborado ex profeso, y el Inventario de Calidad de Vida y Salud. Los resultados se discuten en términos del incremento en el tiempo dedicado a la autoexploración en ambas condiciones, la efectividad relativa de la autoexploración mamaria en ambas condiciones, la identificación diferencial de alteraciones en el modelo de simulación; y el efecto observado en las respuestas al citado inventario.
\end{abstract}

Palabras clave: Procedimiento instruccional; Calidad de vida; Modelos de simulación; Cáncer de mama; Modelo de salud biológica.

\begin{abstract}
Chronic non-communicable diseases are currently the worldwide leading cause of death. In particular, breast cancer is the most common type of cancer among women. Breast care programs have focused on the mass dissemination of information; however, this does not seem to be an effective strategy for actually modifying unhealthy practices. Since the psychological model of biological health allows for the identification of risk factors that contributes to illness development, the aim of the present study was to evaluate an instructional procedure to train in the identification and implementation of instrumental behaviors related to breast care, as well as its effect on quality of life. Through a pretest-posttest design 22 women were randomly assigned to two training conditions, the first included: reading materials, video, images and a model for simulation, the second included reading materials, video, and images only. Two questionnaires were administered: one specifically developed to assess correct responses, and the Quality of Life and Health Inventory. Results are discussed in terms of the increment of time spent in self-examination in both conditions, the relative effectiveness of performing such breast self-examination, the differential identification of alterations on the simulation model, and the effects on quality of life.
\end{abstract}

Key words: Instructional procedure; Quality of life; Simulation models; Breast cancer; Biological health model.

\footnotetext{
${ }^{1}$ Departamento de Psicología y Ciencias de la Comunicación, Universidad de Sonora, Blvd Luis Encinas y Rosales s/n, Col. Centro, 83000 Hermosillo, Son., México, tel.: (666)259-21-73, correos electrónicos: ernestovalenciaps@gmail.com, karla@psicom.uson.mx, irigoyen@psicom.uson.mx y miriam.jimenez@unison.mx. Artículo recibido el 24 de agosto y aceptado el 16 de noviembre de 2017.

Citación: Valencia, J.E., Acuña, K.F., Irigoyen, J.J. y Jiménez, M.Y. (2018). Efectos de un procedimiento instruccional en el desarrollo de habilidades para el cuidado de las mamas. Psicología y Salud, 28(2), 143-154.
} 


\section{INTRODUCCIÓN}

$\mathrm{L}$ as enfermedades crónicas no transmisibles (ECNT en lo sucesivo) son la principal causa de muerte en todo el mundo, con $68 \%$ de defunciones en el año 2012 (Organización Mundial de la Salud [OMS], 2017). Dichas enfermedades son afecciones que progresan lentamente y que tienen una larga duración. Entre las ECNT destacan las enfermedades cardiovasculares (como los infartos al miocardio o los accidentes cerebrovasculares), el cáncer, las enfermedades respiratorias crónicas (como la neumonía obstructiva crónica y el asma) y la diabetes.

En México, según un perfil elaborado por la OMS (2016), se ha visto que existe una probabilidad de $16 \%$ de morir entre los 30 y 70 años de edad a causa de una de las cuatro principales ECNT mencionadas anteriormente. Esto se debe a la continua exposición de la población a ciertos factores de riesgo, como el consumo de tabaco y alcohol, la hipertensión arterial y la obesidad.

En particular, el cáncer es la segunda causa de muerte en los países desarrollados después de las enfermedades cardiovasculares. Los datos epidemiológicos apuntan a que esta tendencia aumente en países menos desarrollados, como México (OMS, 2016). Entre los tipos de cáncer caracterizados por su alta mortalidad se encuentra el de mama (CaMa en lo sucesivo). Según un informe de la American Cancer Society (2016) y la base de datos GLABOCAN (Ferlay et al., 2015), el CaMa es por mucho el tipo de cáncer más común en todo el mundo, y en el año 2012 fue el más frecuente en las mujeres, con un estimado de 1.67 millones de nuevos casos, cifra que representó la cuarta parte de todos los casos.

Aun cuando el cáncer en general y el CaMa en particular son uno de los principales problemas del sistema de salud mexicano, las acciones que se han tomado han resultado poco efectivas e insuficientes, lo que ha quedado demostrado con el continuo aumento de las tasas de incidencia y mortalidad señaladas por el Instituto Nacional de Geografía y Estadística (INEGI) (2016) durante el periodo 2007-2015.

Respecto a las medidas que se deben tomar para el control de esta enfermedad, Anderson y Jakesz (2008), en una revisión de la iniciativa global para la salud de las mamas (BHGI, por sus siglas en inglés), señalan que el mejor procedimiento para la detección temprana y la reducción de la mortalidad por CaMa es la mamografía; sin embargo, no todos los países pueden cubrir a su población con este procedimiento, por lo que el uso de técnicas como la exploración clínica y la autoexploración de mamas resultan ser útiles en naciones con ingresos medios y bajos.

En una caracterización de las medidas tomadas por nuestro país hecha por Uscanga, Torres, Ángeles, Domínguez y Lazcano (2014) han reportado que el tamizaje del CaMa es oportunista; es decir, la realización de la mamografía es una decisión individual, por lo tanto, no hay evidencia alguna de su efectividad en virtud de que este procedimiento no se lleva a cabo, generalmente por corresponder a un programa específico de salud o por la falta de promoción. Además, apuntan que en nuestro país los esfuerzos se han orientado a la adquisición de infraestructura y el establecimiento de relaciones contractuales con la iniciativa privada, sin asegurar previamente el empleo óptimo de la capacidad actualmente instalada.

Otro estudio realizado por López, Torres, López y Rueda (2001) reporta que 90\% de las mujeres reconocen por sí mismas la presencia de alteraciones o signos de CaMa, lo que las lleva a buscar atención médica. Esta es una realidad en nuestro país, donde las acciones que se toman para la detección temprana no son efectivas ni suficientes.

La utilidad de la autoexploración para la detección temprana de CaMa ha sido reportada por diversos autores (Adams et al., 1976; Al-Azmy et al., 2013; Ceber, Turk y Ciceklioglu, 2010; Donmez, Dolgun y Yavuz, 2012, entre muchos otros). De igual modo, investigaciones como la llevada a cabo por Al-Azmy et al. (2013) muestran que, de manera retrospectiva, la autoexploración está asociada a un diagnóstico menos grave de la enfermedad. Dicha autoexploración es una técnica que consiste en una secuencia estructurada de pasos para explorar las zonas más propensas al desarrollo de CaMa (mamas, axila y cuello), lo que permite a quien la lleva a cabo correctamente diferenciar las alteraciones en la mama y el tejido normal. Es por ello que su enseñanza no debe ser tomada a la ligera y que deben desarrollarse estrategias acordes al tipo de habilidad a entrenar. 
Investigaciones como las realizadas por Assaf, Cummings, Graham, Mettlin y Marshall (1985) y Fletcher, O'Malley y Bunce (1985), en las que se muestran diferentes estrategias para enseñar la técnica de autoexploración de mamas, han reportado efectos diferenciales según el tipo de entrenamiento. Las conclusiones de estas pesquisas indican mejores resultados en las participantes que fueron entrenadas con un modelo de simulación, en comparación con aquellas a las que solo se les mostraron materiales informativos. Tal como se señaló, los resultados apuntan a que las estrategias de enseñanza a utilizar deben ser acordes al tipo de habilidad a entrenar; es decir, si lo que se requiere es que el participante realice algún tipo de actividad específica, no es suficiente proporcionarle la información pertinente, sino que además es necesario exponerlo a condiciones que simulen la condición o el escenario en los que debe ejecutarse.

Respecto al tipo de habilidad a entrenar, algunos estudios elaborados en el ámbito de la psicología, y específicamente en el área de la educación (Irigoyen, Jiménez y Acuña, 2016), indican que las estrategias de enseñanza no pueden desarrollarse al margen de la habilidad a entrenar, esto es, que debe existir una relación de pertinencia entre aquello que se enseña y la forma en cómo se enseña. Por consiguiente, las condiciones de evaluación deben diseñarse en función de la habilidad a entrenar. Si lo que se desea es que las personas realicen la autoexploración de mamas, es a partir de la práctica efectiva que esta técnica puede tener sentido para la prevención o la detección temprana de la enfermedad. Es por esta razón que quien enseña debe ser un profesional sumamente capacitado tanto en la técnica de autoexploración, como en el entrenamiento efectivo de la misma.

La Psicología, como la disciplina encargada de estudiar la relación del individuo con su medioambiente funcional, contribuye al ámbito de la salud con aproximaciones que permiten analizar las modulaciones y ajustes del comportamiento de los individuos a las condiciones ambientales o del propio comportamiento, las cuales pueden reducir o incrementar el riesgo de contraer una enfermedad.

El modelo psicológico de la salud biológica elaborado por Ribes (1990) es una aproximación contemporánea al estudio del proceso de salud-enfermedad. En la lógica de este modelo, la salud (en términos biológicos) se concibe como la ausencia de enfermedad o un estado de baja propensión a contraerla, y es consecuencia de la manera en que el individuo establece contacto y se regula ante las condiciones ambientales. Si el comportamiento del individuo es el mediador de los efectos del ambiente sobre su organismo, la prevención de la enfermedad descansa en la disponibilidad de planear y desarrollar formas de comportamiento eficaces para estos fines.

El citado modelo describe los factores relevantes a considerar para el mantenimiento o la recuperación de la salud de los individuos; enfatiza el papel de las competencias y, sobre todo, los diferentes tipos y niveles de las habilidades requeridas ante cada situación específica. De manera paralela, señala acciones oportunas para cada nivel de prevención, tanto para la enfermedad ya diagnosticada, como para aquellas que aún no se presentan (Rodríguez, Rentería, Rosales y Rodríguez, 2014).

Uno de los componentes del modelo que se retoma en el desarrollo de este trabajo es el de las competencias instrumentales, las cuales se definen como acciones de los individuos que, directa o indirectamente, disminuyen o aumentan la probabilidad de que el individuo contraiga una enfermedad. En este sentido, las conductas instrumentales se definen como las acciones efectivas y específicas para prevenir o aumentar los riesgos de contagio de los agentes fisicoquímicos y biológicos productores de la patología biológica.

Como se mencionó, las conductas instrumentales pueden clasificarse de dos maneras: directas, como aquellas que producen el contacto con el agente patógeno, sea este un causante de lesiones o infeccioso, e indirectas, las cuales, sin producir contacto específico con agentes patógenos o dañinos, aumentan la vulnerabilidad del organismo ante la acción de los mismos. En el caso de las ECNT, como el CaMa, se puede decir que su desarrollo e identificación tardía están relacionados principalmente con conductas instrumentales de riesgo indirectas, toda vez que no entran en contacto directo con el desarrollo de este tipo de enfermedades; es decir, tienen que ver con conductas vinculadas a hábitos alimenticios inadecuados, tales como la alta ingesta de alcohol y tabaco, ejercicio físico mal dosificado o técnicas de au- 
toexploración mal practicadas, por solo mencionar algunas.

Un componente adicional, por estar estrechamente relacionado con los objetivos del sistema de salud, así como con las posibilidades de mejora de las personas, es el concepto de calidad de vida, en particular el relacionado con la salud. La dimensión psicológica de la calidad de vida (cf. Moreno y Ximénez, 1996) se fundamenta en las practicas individuales, ya sea como prevención de la enfermedad o como prácticas que favorecen la cura y la rehabilitación, así como aquellas conductas vinculadas a la preservación de los ambientes físicos y sociales que delimitan las prácticas colectivamente compartidas como cultura (educación, trabajo, arte, ciencia, etc.).

Así, Carpio, Pacheco, Flores y Canales (2000) definen la calidad de vida como "el modo en que las distintas condiciones biológicas y culturales se conjugan como los aspectos psicológicos para definir un modo particular de existencia práctica de los individuos concretos; es decir, la calidad de vida puede ser concebida como la resultante funcional del estado conjunto de las condiciones biológicas, socioculturales y psicológicas de los individuos, estado que define el modo en que éstos se ajustan a las situaciones cotidianas en que su comportamiento, como modo de existencia práctica, tiene lugar, así como los resultados que dicho ajuste tiene sobre el ambiente y sobre el propio organismo" (p. 4).

La utilización de un instrumento que mida la calidad de vida referida a la salud y que permita discriminar entre personas con enfermedad crónica o aguda, y personas sanas es fundamental en este tipo de investigaciones; cabe mencionar que el Inventario de Calidad de Vida y Salud (InCaViSa en lo sucesivo), desarrollado por Riveros, Sánchez-Sosa y Del Águila (2009), representa una valiosa contribución que hace posible realizar dicha tarea. Las investigaciones de Riveros, Castro y Lara (2008), Garduño, Riveros y Sanchez-Sosa (2010) y González et al. (2014) muestran la efectividad de este instrumento de evaluación para registrar los cambios en la percepción de las personas durante la exposición a entrenamientos orientados a modificar conductas asociadas con la mejora de la salud.

La necesidad de programas que se enfoquen en la modificación de conductas instrumentales relacionadas con el mantenimiento de la salud, así como de otros que coadyuven en la detección temprana de enfermedades como el CaMa, es cada vez más urgente, conclusión que se desprende de la revisión de las cifras de mortalidad y prevalencia, que cada año son mayores. Considerando lo dicho hasta este momento, el objetivo de este estudio fue evaluar un procedimiento instruccional para el entrenamiento en la identificación e implementación de conductas instrumentales relacionado con el cuidado de las mamas y su efecto sobre en calidad percibida relacionada con la salud.

\section{MÉTODO}

\section{Participantes}

A partir de un muestreo no probabilístico por conveniencia (Kerlinger y Lee, 2001), se seleccionaron 22 mujeres: siete estudiantes universitarias, siete dedicadas al hogar y ocho sobrevivientes de cáncer de mama. Todas las participantes firmaron un formato de consentimiento informado para participar en la investigación. El rango de edad osciló entre 19 y 68 años, siendo 40.8 años la edad promedio. De la muestra, $59 \%$ reportó tener antecedentes familiares de algún tipo de cáncer $\mathrm{y}$, de estas, 69\% señaló tener antecedentes familiares de CaMa.

\section{Diseño}

Se implementó un diseño cuasi experimental pretest-postest con grupo control (Campbell y Stanley, 1978). Las participantes se dividieron aleatoriamente en dos grupos, las cuales fueron expuestas a una de las dos condiciones de entrenamiento que se muestran en la Tabla 1.

\section{Materiales}

Se elaboró un material de apoyo didáctico (Irigoyen y Velázquez, 2016) para ilustrar y modelar el cuidado de las mamas (autoexploración), el cual consta de cuatro módulos y ocho actividades (véase Fig. 1). Dicho material contiene videos ilustrativos para realizar la técnica de autoexploración, así como un registro de cada uno de los pasos para llevar a cabo este procedimiento. 
Tabla 1. Representación del diseño pretest-postest utilizado.

\begin{tabular}{|c|l|l|l|l|}
\hline Grupos & \multicolumn{1}{|c|}{$\begin{array}{c}\text { Momento 1: } \\
\text { Pretest }\end{array}$} & \multicolumn{1}{|c|}{$\begin{array}{c}\text { Momento 2: } \\
\text { Entrenamiento }\end{array}$} & \multicolumn{1}{c|}{$\begin{array}{c}\text { Momento 3: } \\
\text { Postest }\end{array}$} & \multicolumn{1}{c|}{$\begin{array}{c}\text { Momento 4: } \\
\text { Seguimiento }\end{array}$} \\
\hline $\begin{array}{c}\text { Grupo 1 } \\
\mathrm{N}=12\end{array}$ & $\begin{array}{l}\text { Se aplicaron el instrumento de } \\
\text { competencias instrumentales } \\
\text { e InCaViSa }\end{array}$ & $\begin{array}{l}\text { Condición 1: } \\
\text { Videoimágenes } \\
\text { Modelo de simulación }\end{array}$ & $\begin{array}{l}\text { Se aplicaron inventarios de } \\
\text { competencias instrumentales }\end{array}$ & Se aplicó InCaViSa \\
\hline $\begin{array}{c}\text { Grupo 2 } \\
\mathrm{N}=10\end{array}$ & & $\begin{array}{l}\text { Condición 2: } \\
\text { Videomágenes }\end{array}$ & & \\
\hline
\end{tabular}

Figura 1. Ilustración del material utilizado en el entrenamiento.

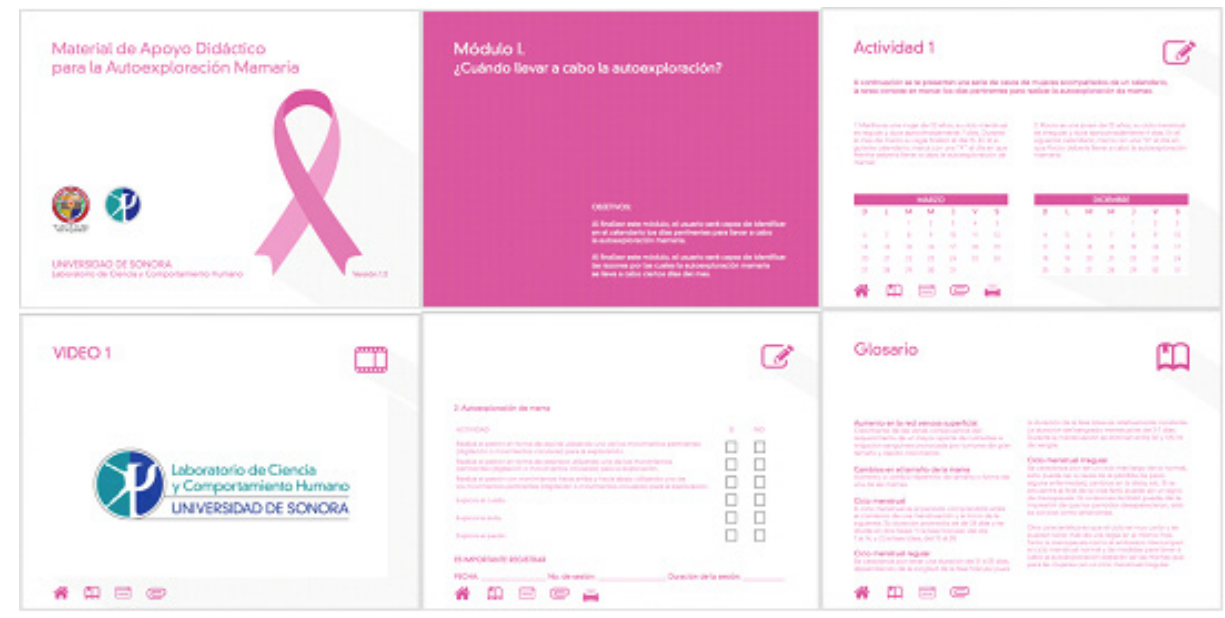

Con el propósito de modelar y registrar la técnica de autoexploración de mamas, se empleó un modelo de simulación del tórax femenino con arnés que contiene ocho diferentes alteraciones asociadas al desarrollo de CaMa.

Figura 2. Modelo de simulación utilizado durante el entrenamiento.

\section{Instrumentos}

Se utilizaron dos instrumentos para la recolección de información, el primero de los cuales fue elaborado ex profeso que tuvo dos objetivos: primeramente, evaluar los conocimientos relacionados con el desarrollo de la enfermedad (CaMa) y los procedimientos para el tratamiento de la enfermedad; en segundo lugar, evaluar las conductas de cuidado de las mamas (autoexploración).

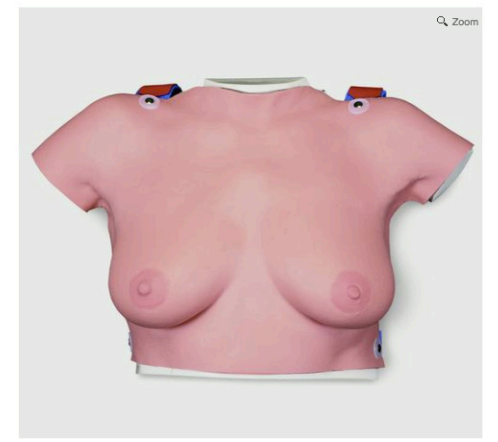

El segundo fue el InCaViSa utilizado para evaluar la percepción del paciente, usuario o sujeto respecto a una serie de situaciones relacionadas con la salud y los comportamientos asociados a esta. Es un instrumento autoaplicable estandarizado que consta de 53 afirmaciones en una escala tipo Likert con seis opciones de respuesta, el cual permite evaluar doce áreas: preocupaciones, desempeño físico, aislamiento, percepción corporal, funciones cognitivas, actitud ante el tratamiento, 
tiempo libre, vida cotidiana, familia, redes sociales, dependencia médica y relación con el médico.

\section{Procedimiento}

A todos los participantes se les explicó en qué consistía el programa de CaMa y la autoexploración, subrayando la importancia de su comportamiento en el desarrollo del CaMa y los beneficios potenciales de participar en un programa como este. Se les presentó y aclaró asimismo el propósito del estudio, sus potenciales beneficios, la garantía de anonimato y confidencialidad, y la posibilidad de abandonar la investigación en cualquier momento sin consecuencia alguna.

Una vez firmado el formato de consentimiento informado, se asignó aleatoriamente a las participantes en una de las dos condiciones para el entrenamiento, consistente la primera en la exposición de textos, videos y un modelo de simulación, y la segunda únicamente en materiales de texto y video (véase Tabla 1).

Se trabajó con las participantes en cuatro momentos:

Momento 1: Se citó a las participantes para llevar a cabo el programa CaMa y la autoexploración. En esta primera sesión se aplicaron los instrumentos InCaVisa y el cuestionario ex profeso. La sesión se llevó a cabo en una sala libre de ruido y tuvo una duración de 30 minutos, aproximadamente. Una vez que terminaron de contestar los instrumentos, se les citaba para llevar el taller de autoexploración y CaMa.

Momento 2: Se llevó a cabo el entrenamiento, que por motivos didácticos se dividió en dos módulos: el primero contenía información referente al cáncer en general y al CaMa en particular, y el segundo información sobre cómo y cuándo llevar a cabo la autoexploración mamaria. Con el propósito de monitorear la ejecución de las participantes y la efectividad del programa, al concluir cada módulo las participantes respondían diversas preguntas referidas al contenido del mismo.

La secuencia del entrenamiento fue la siguiente: 1) Las participantes se colocaban el modelo de simulación y realizaban la autoexploración, y al concluir se les preguntaba si habían podido palpar u observar alguna modificación en la mama. 2) Se les exponía al material con video, imágenes y modelamiento, o video e imágenes, según la condición (ambas modalidades del taller tuvieron una duración aproximada de dos horas). 3) Hecho lo anterior, se colocaban nuevamente el modelo de simulación para realizar la autoexploración, y de igual manera se les preguntaba al concluir si habían podido identificar alguna modificación en el modelo.

Momento 3: Nuevamente se les solicitaba que contestaran los instrumentos InCaViSa y el instrumento elaborado ex profeso. Lo anterior se llevó a cabo en una sala sin ruido y tuvo una duración aproximada de 30 minutos.

Momento 4: Un mes después se citó a las participantes. En esta última sesión se aplicaron a las participantes los instrumentos InCaViSa y el cuestionario ex profeso, los cuales fueron respondidos bajo las mismas condiciones que en los momentos anteriores.

\section{RESULTADOS}

Los resultados de la identificación de las características del cáncer, los signos y métodos del CaMa y la prueba de conocimientos mostraron porcentajes altos durante el pretest y el postest en ambas condiciones (Tabla 2).

Tabla 2. Porcentaje de aciertos en la evaluación de conocimientos.

\begin{tabular}{|l|c|c|c|c|}
\hline \multirow{2}{*}{ Participantes } & \multicolumn{2}{|c|}{ Condición 1 } & \multicolumn{2}{c|}{ Condición 2 } \\
\cline { 2 - 5 } & Pre & Post & Pre & Post \\
\hline Estudiante & $85.6 \%$ & $74.4 \%$ & $79.2 \%$ & $89.2 \%$ \\
\hline Ama de casa & $81.9 \%$ & $88.1 \%$ & $90.8 \%$ & $86.7 \%$ \\
\hline Sobreviviente & $88.1 \%$ & $85.0 \%$ & $93.1 \%$ & $92.5 \%$ \\
\hline Total & $85.2 \%$ & $82.5 \%$ & $88.3 \%$ & $89.8 \%$ \\
\hline
\end{tabular}


Tales resultados parecen indicar, al menos para esta muestra, que las participantes pudieron identificar la información pertinente para el tipo de enfermedad; es decir, conocían las características básicas de la misma, el cómo se trata y las medidas que se deben tomar una vez diagnosticada.

El análisis de conocimientos (características del cáncer, signos del CaMa y métodos de detección temprana) no mostró diferencias estadísticamente significativas $(p=.525)$ a un nivel de significancia de .05 . Tal resultado puede atribuirse a que las personas no tienen dificultades con el decir y el hacer, esto es, los sujetos de ambas condiciones pudieron identificar elementos discursivos relacionados con el desarrollo de la enfermedad, la forma en que se trata y las medidas que se toman una vez detectada.

Como parte de la evaluación de habilidades instrumentales, se evaluó la correspondencia entre el reportar conocer los pasos de la autoexploración y llevarla a cabo de manera regular (Tabla 3).

Tabla 3. Porcentaje de correspondencia entre decir y hacer respecto a la autoexploración de mamas.

\begin{tabular}{|c|c|c|c|c|}
\hline \multirow[t]{2}{*}{ Correspondencia } & \multicolumn{4}{|c|}{$\begin{array}{l}\text { Preguntas evaluadas: } \\
\text { 1) ¿Conoces los pasos para realizar la autoexploración? } \\
\text { 2) ¿Llevas a cabo la autoexploración de mamas? }\end{array}$} \\
\hline & Estudiante $(n=7)$ & Ama de casa $(n=7)$ & Sobreviviente $(n=8)$ & Total \\
\hline Positiva & $1(14.3 \%)$ & $3(42.9 \%)$ & $2(25.0 \%)$ & $6(27.0 \%)$ \\
\hline Negativa & $4(57.1 \%)$ & $2(28.6 \%)$ & $3(37.5 \%)$ & $9(40.9 \%)$ \\
\hline No correspondencia & $2(28.6 \%)$ & $2(28.6 \%)$ & $3(37.5 \%)$ & $7(31.8 \%)$ \\
\hline
\end{tabular}

Positiva: cuando la participante contesta positivamente ambas preguntas.

Negativa: cuando la participante contesta negativamente ambas preguntas.

No correspondencia: cuando la participante contesta afirmativamente solo una de las preguntas.

Como se puede observar, no hubo correspondencia entre el decir y el hacer en cuanto a la autoexploración mamaria. Si bien las participantes señalaron saber qué es el cáncer, cómo se contrae, cómo se trata y cuáles son las medidas de prevención, no ponían en práctica dichas conductas de prevención. Lo anterior apunta al hecho de que "el decir del hacer" no es suficiente para que este último se lleve a cabo, y menos aún se realice de manera apropiada.

Adicionalmente, se pidió a las participantes simular el procedimiento de autoexploración sobre el modelo de simulación (Figura 3).

Figura 3. Se ilustra la localización de las alteraciones presentadas en el modelo de simulación.

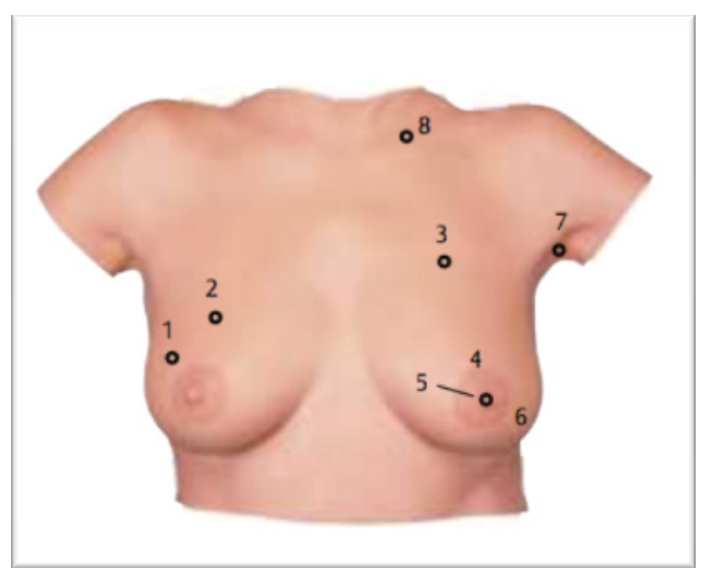

Este modelo contenía ocho alteraciones que debían ser identificadas y que se muestran en la Tabla 4 . En ella se observa el número de la participante (y el porcentaje) que identificó la alteración; se aprecia que las participantes de la condición 1 lograron identificar un mayor número de alteraciones en los resultados posteriores al entrenamiento. Las participantes de ambas condiciones 
tuvieron especial dificultad para identificar las alteraciones 5 (retracción del pezón), 6 (piel de naranja), 7 (alteración en la axila) y 8 (alteración en el cuello). Tales dificultades pueden deberse a las características físicas del modelo de simulación (por ejemplo, la falta de correspondencia corporal con la de la participante).

Tabla 4. Número de participantes que identificaron alteraciones sobre el modelo de simulación.

\begin{tabular}{|c|c|c|c|c|c|}
\hline \multirow[t]{2}{*}{ Alteración } & \multirow[t]{2}{*}{ DESCRIPCIÓN DE LAALTERACIÓN } & \multicolumn{2}{|c|}{$\begin{array}{c}\text { Condición } 1 \\
(\mathrm{n}=12)\end{array}$} & \multicolumn{2}{|c|}{$\begin{array}{l}\text { Condición } 2 \\
(\mathbf{n}=10)\end{array}$} \\
\hline & & Pre & Post & Pre & Post \\
\hline 1 & $\begin{array}{l}\text { Tumor redondo, movible, profundidad } 10 \mathrm{~mm} \text {, diámetro } 20 \mathrm{~mm} \text {, } \\
\text { probablemente benigno. }\end{array}$ & $\begin{array}{c}1 \\
(8.3 \%)\end{array}$ & $\begin{array}{c}5 \\
(41.7 \%)\end{array}$ & $\begin{array}{c}1 \\
(10.0 \%)\end{array}$ & $\begin{array}{c}4 \\
(40.0 \%)\end{array}$ \\
\hline 2 & $\begin{array}{l}\text { Tumor redondo, movible, profundidad } 5 \mathrm{~mm} \text {, diámetro } 20 \mathrm{~mm} \text {, } \\
\text { probablemente benigno. }\end{array}$ & $\begin{array}{c}0 \\
(0.0 \%)\end{array}$ & $\begin{array}{c}9 \\
(75.0 \%)\end{array}$ & $\begin{array}{c}1 \\
(10.0 \%)\end{array}$ & $\begin{array}{c}5 \\
(50.0 \%)\end{array}$ \\
\hline 3 & $\begin{array}{l}\text { Tumor irregular, adherido, profundidad } 5 \mathrm{~mm} \text {, diámetro } \\
\text { aproximado } 35 \times 25 \mathrm{~mm} \text {, probablemente maligno. }\end{array}$ & $\begin{array}{c}1 \\
(8.3 \%)\end{array}$ & $\begin{array}{c}9 \\
(75.0 \%)\end{array}$ & $\begin{array}{c}0 \\
(0.0 \%)\end{array}$ & $\begin{array}{c}6 \\
(60.0 \%)\end{array}$ \\
\hline 4 & $\begin{array}{l}\text { Tumor con forma de columna, irregular, adherido, profundidad } \\
5 \mathrm{~mm} \text {, diámetro aproximado } 30 \mathrm{~mm} \text {, maligno. }\end{array}$ & $\begin{array}{c}2 \\
(16.7)\end{array}$ & $\begin{array}{c}11 \\
(91.7 \%) \\
\end{array}$ & $\begin{array}{c}0 \\
(0.0 \%) \\
\end{array}$ & $\begin{array}{c}5 \\
(50.0 \%) \\
\end{array}$ \\
\hline 5 & $\begin{array}{l}\text { Retracción permanente del pezón (primera presentación), } \\
\text { a menudo relacionada con tumor maligno. }\end{array}$ & $\begin{array}{c}0 \\
(0.0 \%)\end{array}$ & $\begin{array}{c}0 \\
(0.0 \%) \\
\end{array}$ & $\begin{array}{c}0 \\
(0.0 \%) \\
\end{array}$ & $\begin{array}{c}0 \\
(0.0 \%)\end{array}$ \\
\hline 6 & $\begin{array}{l}\text { Piel con estructura de "piel de naranja", con retracción } \\
\text { de los poros como consecuencia de la estasis linfática } \\
\text { en los tumores malignos. }\end{array}$ & $\begin{array}{c}1 \\
(8.3 \%)\end{array}$ & $\begin{array}{c}2 \\
(16.7 \%)\end{array}$ & $\begin{array}{c}0 \\
(0.0 \%)\end{array}$ & $\begin{array}{c}0 \\
(0.0 \%)\end{array}$ \\
\hline 7 & $\begin{array}{l}\text { Ganglio linfático irregular, fuertemente adherido, } \\
\text { profundidad } 10 \mathrm{~mm} \text {, diámetro aproximado } 35 \times 25 \mathrm{~mm} \text {, } \\
\text { probablemente maligno. }\end{array}$ & $\begin{array}{c}0 \\
(0.0 \%)\end{array}$ & $\begin{array}{c}4 \\
(33.3 \%)\end{array}$ & $\begin{array}{c}0 \\
(0.0 \%)\end{array}$ & $\begin{array}{c}0 \\
(0.0 \%)\end{array}$ \\
\hline 8 & $\begin{array}{l}\text { Tumor de } 5 \mathrm{~mm} \text { de profundidad y } 20 \mathrm{~mm} \text { de diámetro. } \\
\text { Maligno. }\end{array}$ & $\begin{array}{c}0 \\
(0.0 \%)\end{array}$ & $\begin{array}{c}1 \\
(8.3 \%)\end{array}$ & $\begin{array}{c}0 \\
(0.0 \%)\end{array}$ & $\begin{array}{c}2 \\
(20.0 \%)\end{array}$ \\
\hline
\end{tabular}

Nota: Las descripciones de las alteraciones en el modelo de simulación son especificadas por el proveedor del producto: 3B Scientific ${ }^{\circledR}$

De manera conjunta, se evaluó el tiempo total de la autoexploración. Como se puede observar en las Gráficas 1 y 2, las participantes de ambas condiciones aumentaron considerablemente el tiempo de exploración durante el postest, pasando de un promedio de 90.2 segundos en el pretest a 348.3 segundos en el postest en la condición 1, y de 73.1 segundos en el pretest a 321.8 segundos en el postest en la condición 2.

Gráfica 1. Tiempo total de exploración de los participantes en la condición 1.

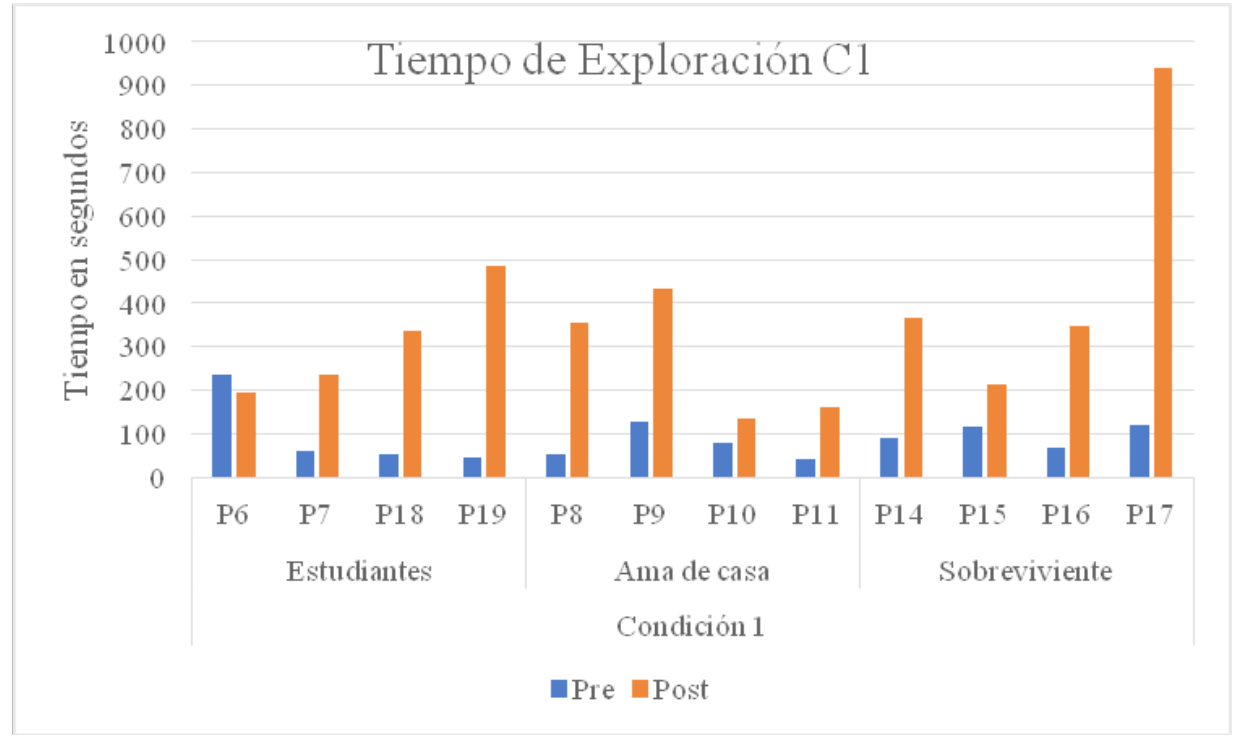


Gráfica 2. Tiempo total de exploración de los participantes en la condición 2.

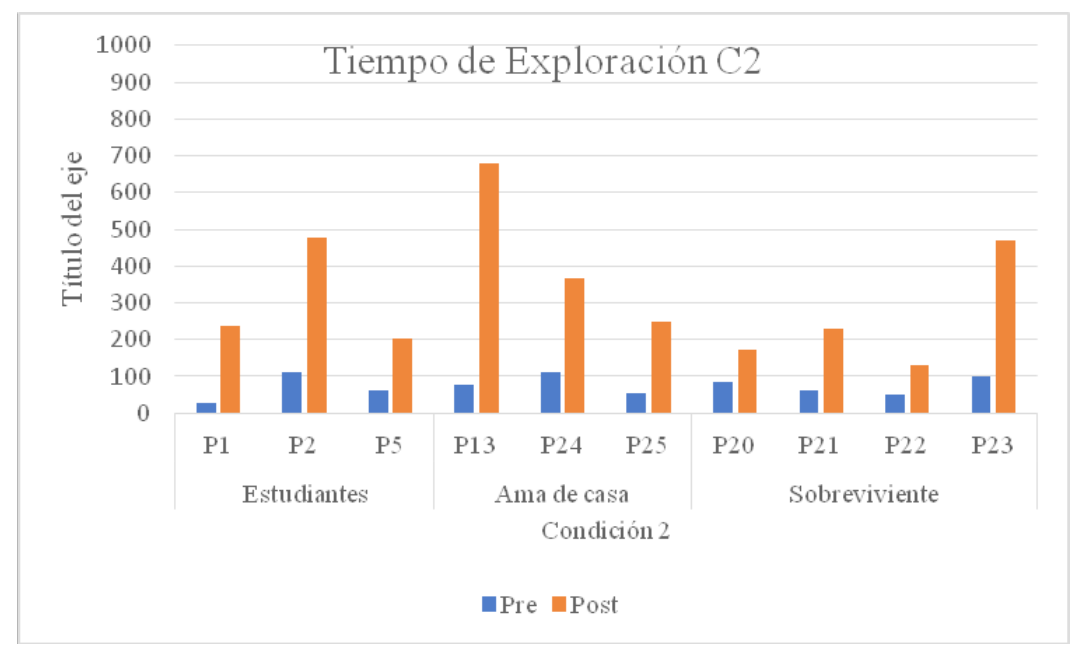

Si bien los resultados anteriores muestran diferencias considerables respecto al pretest, no bastan para hablar de la efectividad de la autoexploración. Los resultados que se muestran en la Tabla 5 indican que la mitad de las participantes expuestas a la condición 1 (video, imágenes y modelo) logran llevar a cabo los pasos para realizar la autoexploración de manera apropiada en el postest, mientras que solo la quinta parte de las participantes de la condición 2 lograron hacerlo correctamente.

Tabla 5. Número de participantes que completaron correctamente la autoexploración.

\begin{tabular}{|l|c|c|c|c|}
\hline \multirow{2}{*}{ Participantes } & \multicolumn{2}{|c|}{$\begin{array}{c}\text { Condición 1 } \\
(\mathbf{n}=\mathbf{1 2})\end{array}$} & \multicolumn{2}{c|}{$\begin{array}{c}\text { Condición 2 } \\
(\mathbf{n}=\mathbf{1 0})\end{array}$} \\
\cline { 2 - 5 } & Pre & Post & Pre & Post \\
\hline \multirow{2}{*}{ Estudiante } & 0 & 1 & 0 & 0 \\
$(0.0 \%)$ & $(8.3 \%)$ & $(0.0 \%)$ & $(0.0 \%)$ \\
\hline Ama de casa & 0 & 1 & 0 & 1 \\
$(0.0 \%)$ & $(8.3 \%)$ & $(0.0 \%)$ & $(10.0 \%)$ \\
\hline Sobreviviente & 0 & 4 & 0 & 1 \\
$(0.0 \%)$ & $(33.3 \%)$ & $(0.0 \%)$ & $(10.0 \%)$ \\
\hline Total & 0 & 6 & 0 & 2 \\
& $(0.0 \%)$ & $(50.0 \%)$ & $(0.0 \%)$ & $(20.0 \%)$ \\
\hline
\end{tabular}

Para el análisis de la calidad de vida se aplicó el InCaViSa en dos momentos: al comienzo de la investigación y un mes después de haber concluido la intervención. Los resultados de únicamente 12 de las 22 participantes (nueve de la condición 1, y tres de la condición 2) se presentan en porcentajes acumulados debido a que no fue posible establecer contacto nuevamente con las participantes.

Los resultados del instrumento InCaViSa se muestran a continuación. La Gráfica 3 muestra una dirección positiva; es decir, a mayor puntaje, mejor percepción de la calidad de vida. Mientras que la Gráfica 4 muestra una dirección negativa; o sea, a mayor puntaje, menor percepción de la calidad de vida. De manera general, en ambas figuras se puede apreciar una ligera mejoría respecto al pretest en las dos condiciones; sin embargo, el alto índice de deserción imposibilita la comparación entre ambas condiciones de entrenamiento en cuanto a la calidad de vida relacionada con la salud. 
Gráfica 3. Resultado de InCaVisa. Categorías de mejora de la calidad de vida.

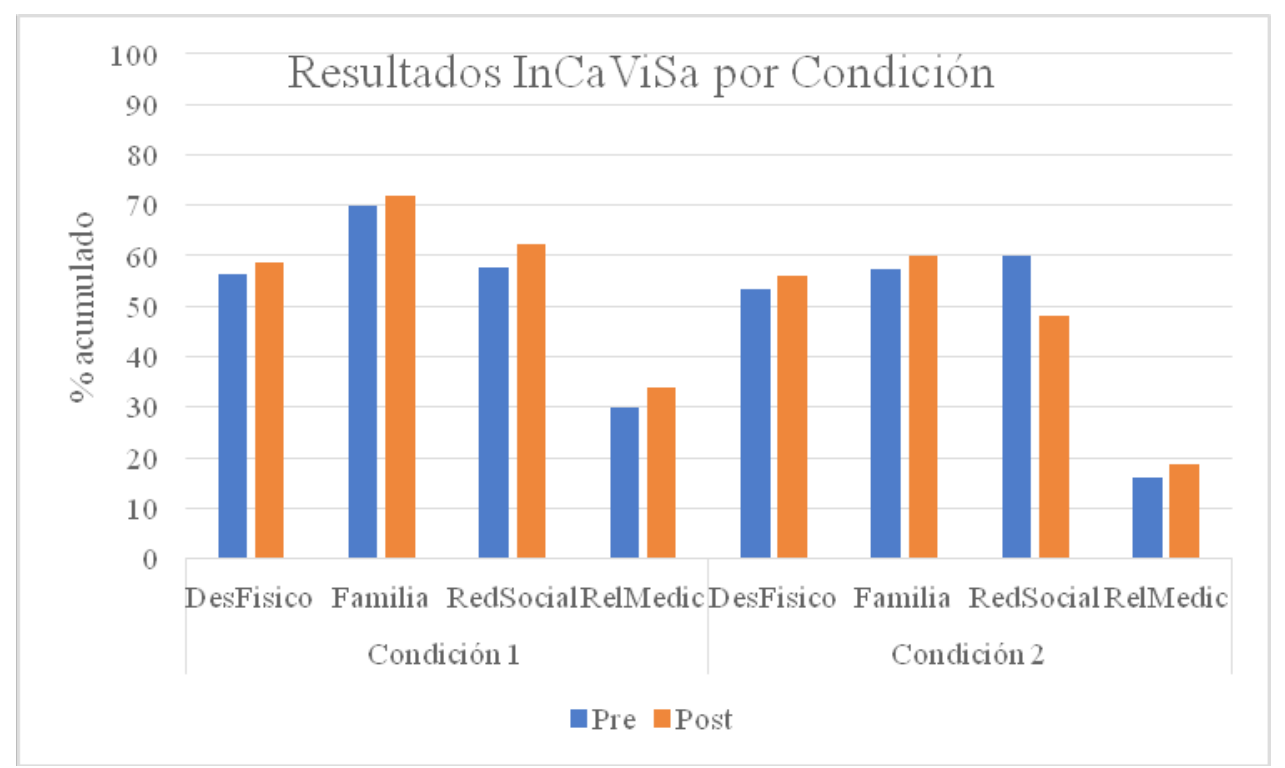

Nota: Desfísico: Desempeño físico; Familia: Familia; RedSocial: Redes sociales; RelMedic: Relación con el médico.

Gráfica 4. Resultados InCaVisa. Categorías de deterioro de la calidad de vida.

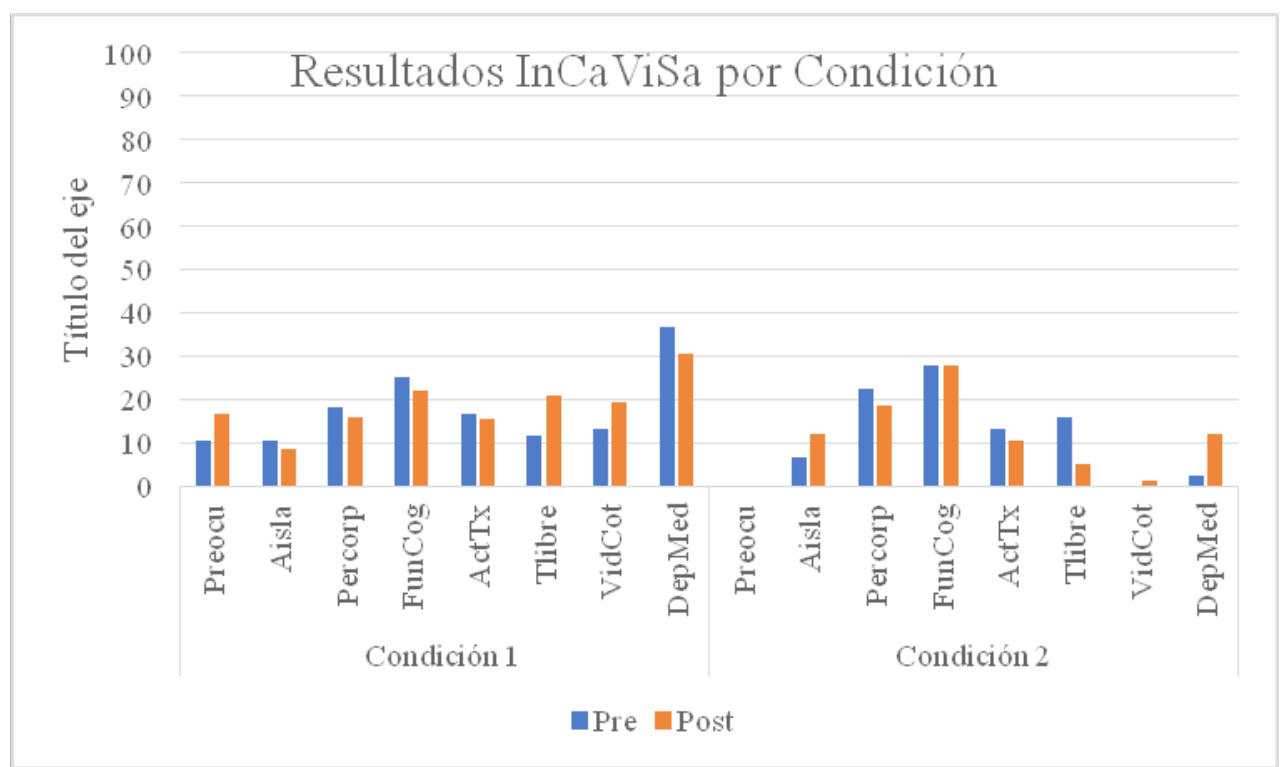

Nota: Preocu: Preocupaciones; Aisla: Aislamiento; Percorp: Percepción corporal; FunCog: Funciones cognitivas; ActTx: Actitud ante el tratamiento; Tlibre: Tiempo libre; VidCot: Vida cotidiana; DepMed: Dependencia médica.

\section{DISCUSIÓN}

El objetivo de este estudio fue evaluar un procedimiento instruccional para el entrenamiento en la identificación e implementación de conductas instrumentales relacionadas con el cuidado de las mamas y su efecto sobre la calidad de vida. Los datos parecen apuntar a una mayor efectividad (en la ejecución de la autoexploración) en aquellas participantes expuestas a la condición en la que se estuvo en contacto con el modelo de simulación. Por el contrario, no parece haber diferencias entre lo que las participantes "conocen" respecto al CaMa, cómo se desarrolla, cómo se trata y cómo se previene.

A juicio de los presentes autores, este trabajo constituye un esfuerzo por mostrar que el desarrollo de competencias instrumentales requiere de 
una enseñanza explicita a través del modelamiento y el moldeamiento que emulen las condiciones reales a las que las personas se ven expuestas en su vida diaria.

Así, el uso de modelos de simulación como el empleado en este estudio sugiere una efectividad relativa respecto al desarrollo de dichas competencias vinculadas con la prevención del CaMa; asimismo, los resultados de la investigación discuten las formas en que las campañas de prevención y promoción de la salud se imparten respecto al cuidado de las mamas, en particular la forma en que se pretende promover habilidades de tipo instrumental, en las que la mera distribución de información no es suficiente para modificar o desarrollar este tipo de habilidades. Para su enseñanza, el entrenamiento debe hacerse por medio de un instructor del área de la salud debidamente entrenado que corrija en el momento los errores cometidos y modele en su caso las maneras adecuadas de practicarse. Toda vez que la identificación de las alteraciones en la mama es lo que importa durante la autoexploración, cada participante debe tener la oportunidad de practicar esta técnica de manera eficiente. Es ahí cuando el empleo de modelos de simulación resulta sumamente útil en virtud de sus características.

Los resultados obtenidos en este estudio parecen coincidir con los de otras investigaciones (Adams et al., 1976; Al-Azmy et al., 2013; Donmez et al., 2012; Hall, Goldstein y Stein, 1977) en las que se muestra que las mujeres que reportan conocer el procedimiento de autoexploración en su mayoría no lo realizan; que aquellas que practican la autoexploración lo hacen incorrectamente, y que es posible mejorar el desempeño referente a las prácticas instrumentales como la autoexploración en términos de una reducción del tiempo requerido, una ejecución adecuada y una mayor sen- sibilidad para detectar alteraciones al momento de implementar modelos de simulación que hagan posible el ejercicio de la conducta a desempeñar.

Las limitaciones de este estudio pueden describirse en términos de 1) el número de observaciones hechas. Dado el tipo de diseño (pre-postest), solamente es posible tener dos mediciones: una al inicio y otra al final del estudio, por lo que no se cuenta con información referente al mantenimiento de las conductas aprendidas; 2) el número de sesiones del entrenamiento. Tal como se señaló, la práctica continua de habilidades posibilita un mejor ajuste a los requerimientos de la tarea, por lo que incluir un mayor número de sesiones de entrenamiento puede mejorar el desempeño de las participantes; 3) el modelo de simulación, que si bien es un material de apoyo sumamente útil para el entrenamiento de este tipo de habilidades, sus características físicas dificultan en ciertas ocasiones el desempeño efectivo de las participantes, y 4) el alto índice de deserción del estudio, por lo cual se recomienda en futuras investigaciones poner en práctica algunas estrategias que mantengan a las participantes a lo largo de todo el estudio, como establecer un sistema de recompensas por asistir a las sesiones de entrenamiento o seguimiento.

Las consideraciones para investigaciones futuras apuntan hacia el diseño de intervenciones que generen un impacto de mayor espectro, expandiéndose así a otras áreas vinculadas al aumento de la calidad de vida de las participantes. Además, se recomiendan las evaluaciones periódicas mensuales y durante un periodo mayor que las llevadas a cabo en este estudio, con la finalidad de evaluar los posibles efectos de este tipo de intervenciones sobre la calidad de vida, el mantenimiento de las conductas prosaludables y la posibilidad de generar una verdadera tecnología educativa aplicable al ámbito de la salud.

\section{REFERENCIAS}

Adams, C.K., Hall, D.C., Pennypacker, H.S., Goldstein, M.K., Hench, L.L., Madden, M.C. y Catania, A.C. (1976). Lump detection in simulated human breasts. Perception \& Psychophysics, 20(3), 163-167.

Al-Azmy, S.F., Alkhabbaz, A., Almutawa, H.A., Ismaiel, A.E., Makboul, G. y El-Shazly, M.K. (2013). Practicing breast self-examination among women attending primary health care in Kuwait. Alexandria Journal of Medicine, 49(3), 281-286.

American Cancer Society (2016). Cancer facts and figures 2016. Recuperado de https://www.cancer.org/es/cancer/cancer-de-seno.html.

Anderson, B.O. y Jakesz, R. (2008). Breast cancer issues in developing countries: An overview of the Breast Health Global Initiative. World Journal of Surgery, 32(12), 2578-2585. 
Assaf, A.R., Cummings, K.M., Graham, S., Mettlin, C. y Marshall, J.R. (1985). Comparison of three methods of teaching women how to perform breast self-examination. Health Education Quarterly, 12(3), 259-272.

Campbell, D. y Stanley, J. (1978). Diseños experimentales y cuasiexperimentales en la investigación social. Buenos Aires: Amorrortu Editores.

Carpio, C., Pacheco, V., Flores, C. y Canales, C. (2000). Calidad de vida: un análisis de su dimensión psicológica. Revista Sonorense de Psicología, 14(1), 3-15.

Ceber, E., Turk, M. y Ciceklioglu, M. (2010). The effects of an educational program on knowledge of breast cancer, early detection practices and health beliefs of nurses and midwives. Journal of Clinical Nursing, 19(15-16), 2363-2371.

Donmez, Y.C., Dolgun, E. y Yavuz, M. (2012). Breast self-examination practices and the effect of a planned training program in western turkey. Asian Pacific Journal of Cancer Prevention, 13(12), 6159-6161.

Ferlay, J., Soerjomataram, I., Dikshit, R., Eser, S., Mathers, C., Rebelo, M. y Bray, F. (2015). Cancer incidence and mortality worldwide: sources, methods and major patterns in GLOBOCAN 2012. International Journal of Cancer, 136(5), E359-E386.

Fletcher, S.W., O’Malley, M.S. y Bunce, L.A. (1985). Physicians' abilities to detect lumps in silicone breast models. The Journal of the American Medical Association, 253(15), 2224-2228.

Garduño, C., Riveros, A. y Sánchez-Sosa, J.J. (2010). Calidad de vida y cáncer de mama: efectos de una intervención cognitivo-conductual. Revista Latinoamericana de Medicina Conductual, 1(1), 69-80.

González R., E., Riveros, A., Benjet, C., González F., C., León H., S.R. y Rico M., G. (2014). Calidad de vida en pacientes con tumores óseos, una comparación entre diferentes tratamientos. Revista de Investigación Clínica, 66(2), 121-128.

Hall, D.C., Goldstein, M.K. y Stein, G.H. (1977). Progress in manual breast examination. Cancer, 40(1), 364-370.

Instituto Nacional de Estadística y Geografía (2016). Estadísticas a propósito del Día Mundial de la Lucha contra el Cáncer de Mama. México: INEGI. Recuperado de: http://www.inegi.org.mx/saladeprensa/aproposito/2015/mama0.pdf.

Irigoyen, J.J. y Velázquez, C. (2016). Material de apoyo didáctico para la autoexploración mamaria (Primera parte). Hermosillo: Universidad de Sonora/Qartuppi.

Irigoyen, J.J., Jiménez, M. y Acuña, K.F. (2016). Discurso didáctico e interacciones sustitutivas en la enseñanza de la ciencia. Enseñanza e Investigación en Psicología, 21(1), 68-77.

Kerlinger, F. y Lee, H.B. (2001). Investigación del comportamiento. México: McGraw-Hill.

López C., L., Torres S., L., López C., M. y Rueda N., C. (2001). Identificación de lesiones mamarias malignas en México. Salud Publica de México, 43(3), 199-202.

Moreno, B. y Ximénez, C. (1996). Evaluación de la calidad de vida. En G. Buela-Casal, V. E. Caballo y J. C. Sierra (Eds.): Manual de evaluación en psicología clínica y de la salud (pp. 1045-1070). Madrid: Siglo Veintiuno.

Organización Mundial de la Salud (2014). Enfermedades no transmisibles: perfiles de México. Ginebra: OMS. Recuperado de: http://www.who.int/nmh/countries/mex_es.pdf.

Organización Mundial de la Salud (2016). Cáncer de mama: prevención y control. Ginebra: OMS. Recuperado de: http://www. who.int/topics/cancer/breastcancer/es/.

Organización Mundial de la Salud (2017). Enfermedades no transmisibles. Ginebra: OMS. Recuperado de: http://www.who.int/ mediacentre/factsheets/fs355/es/.

Ribes, E. (1990). Psicología y salud: un análisis conceptual. Barcelona: Martínez Roca.

Riveros, A., Castro, C. y Lara T., H. (2008). Características de la calidad de vida en enfermos crónicos y agudos. Revista Latinoamericana de Psicología, 41(2), 291-304.

Riveros, A., Sánchez-Sosa, J.J. y Del Águila, M. (2009). Inventario de Calidad de Vida y Salud (InCaViSa). México: El Manual Moderno.

Rodríguez C., M.L., Rentería R., A., Rosales A., A. y Rodríguez S., N.Y. (2014). Propuesta educativa para la salud del paciente con diabetes mellitus tipo 2. Pensando Psicología, 10(17). Recuperado de https://doi.org/10.16925/pe.v10i17.791.

Uscanga S., S., Torres M., G., Ángeles L., A., Domínguez M., R. y Lazcano P., E. (2014). Indicadores del proceso de tamizaje de cáncer de mama en México: un estudio de caso. Salud Publica de México, 56(5), 528-537. 